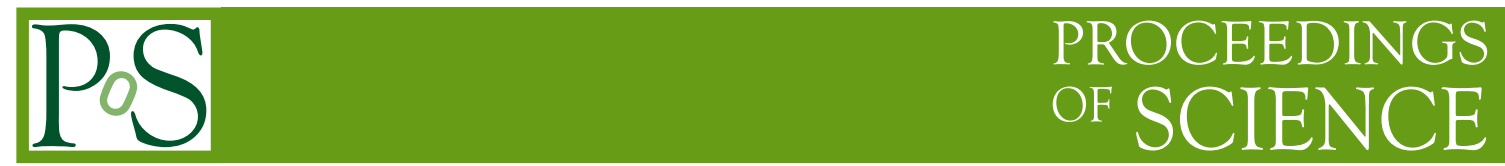

\title{
The European Strategy for Particle Physics and its Update in 2020
}

\author{
Manfred Jeitler* \\ Insitute of High Energy Physics of the Austrian Academy of Sciences \\ E-mail: manfred.jeitlerecern.ch
}

\begin{abstract}
The European Strategy for Particle Physics is the cornerstone of Europe's decision-making process for the long-term future of the field. Currently work is underway to prepare an update of this strategy in 2020 to define the lines along which particle physics in Europe will develop over the coming years.
\end{abstract}

ALPS 2019 An Alpine LHC Physics Summit

April 22 - 27, 2019

Obergurgl, Austria

${ }^{*}$ Speaker. 


\section{What is the "European Strategy for Particle Physics"?}

The European Strategy for Particle Physics is the cornerstone of Europe's decision-making process for the long-term future of the field. Mandated by the CERN Council, it is formed through a broad consultation of the grass-roots particle physics community, it actively solicits the opinions of physicists from around the world, and it is developed in close coordination with similar processes in the US and Japan to ensure coordination between regions and optimal use of resources globally [3].

The decision making body of the "European Strategy" is the CERN Council. Drafting the Strategy Update is the task of the European Strategy Group (ESG). Scientific input to the Strategy is being delivered by the Physics Preparatory Group (PPG). The coordinating body for this process is the Strategy Update Secretariat (SUS).

The European Strategy for Particle Physics was originally initiated in 2006 [1] and first updated in 2012 [2].

\section{The 2020 European Particle Physics Strategy Update (EPPSU)}

The Strategy is due to be updated again by May 2020 to guide the direction of the field into the mid-2020s and beyond [4]. The Secretariat of the European Strategy Group called upon the particle physics community to submit written input by the end of 2018 to prepare the discussions on the Strategy Update which are now taking place in 2019.

By the end of 2018, about 160 contributions had been submitted [5]. So-called "National Road Maps" were submitted by many European countries but also by the USA, Canada, Russia and Japan. In addition, groups representing large projects such as ILC, CLIC, FCCee/FCChh and $\mathrm{CEPC/SppC} \mathrm{but} \mathrm{also} \mathrm{smaller} \mathrm{projects} \mathrm{or} \mathrm{individual} \mathrm{laboratories} \mathrm{submitted} \mathrm{input.}$

Fig.1 highlights the main steps defined to arrive at a strategy update. In 2019, the Open Symposium in Granada [6] took place soon after the ALPS conference and offered a venue to discuss in detail the input provided by the various countries and individual collaborations.

\section{Future Colliders}

As new colliders at the energy frontier are among the most expensive projects in particle physics, the discussion around them is one of the main topics in the whole strategy update process. While the preceding update discussion [2] centered on the High-Luminosity LHC (HL-LHC), now there are competing proposals giving rise to heated discussions. There is widespread consensus that an electron-positron collider above the Higgs production energy is needed for precision measurements of the Standard Model including the Higgs mechanism, which could also unveil hints towards "New Physics" in case of clear deviations of measurement results from Standard Model calculations. For "direct discoveries" not at the "precision frontier" but at the "energy frontier", hadron colliders would probably be required. Such projects envisage not only proton-proton operation but also experiments using ion and electron-proton collisions. 


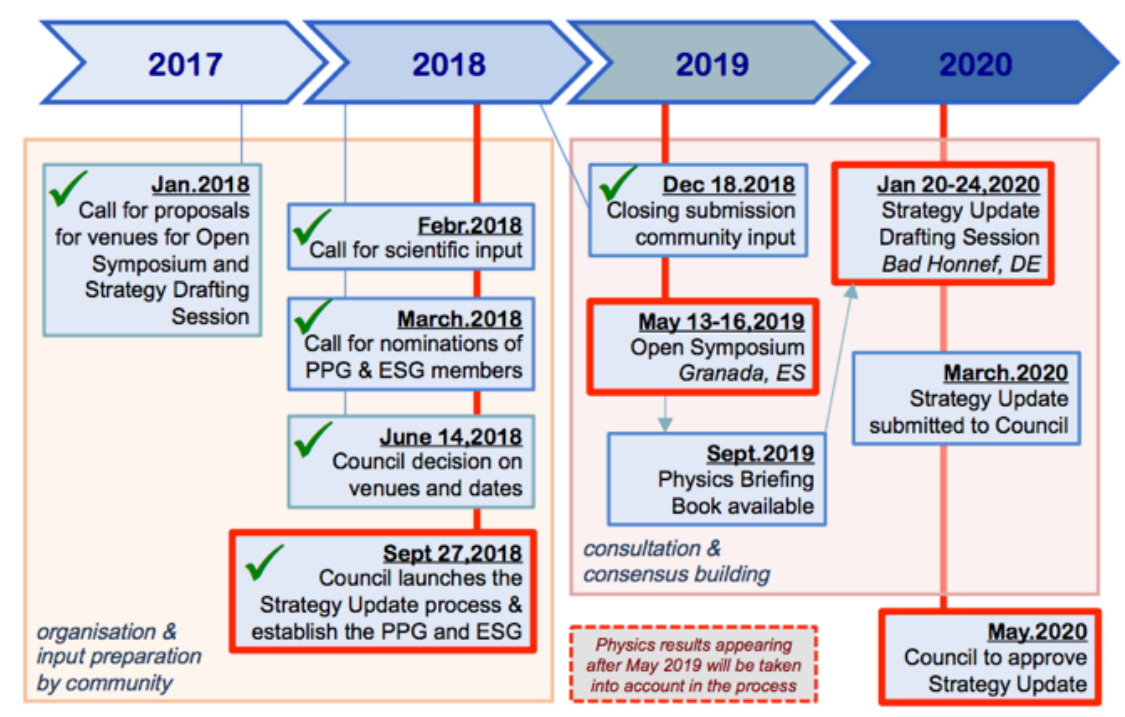

Figure 1: Strategy Update Timeline

\subsection{Electron-positron colliders}

\subsubsection{Linear colliders}

Due to the high synchrotron losses at LEP and in all circular high-energy electron-positron colliders, linear colliders have long been discussed as alternatives. Other frequently quoted arguments in favor of linear colliders are the availability of polarized particle beams and the fact that a linear collider's energy reach can in principle be increased by extending its tunnel while changing the radius is not an option for an existing circular collider.

The ILC ("International Linear Collider") is based on an acceleration scheme using superconducting cavities while CERN's "CLIC" ("Compact Linear Collider") project aims at even higher acceleration gradients and also higher collision energy by using a drive beam rather than klystrons. Shortly before this Conference, both projects were discussed and reviewed in detail at a "Linear Collider Community Meeting" in Lausanne [7].

The ILC Technical Design Report compiled in 2013 foresaw a collision energy of $500 \mathrm{GeV}$ and a price tag of US $\$ 8$ billion. The project was subsequently "re-baselined" (meaning "downscaled") to a collision energy of $250 \mathrm{GeV}$ at 40 percent lower price [8]. The reasoning behind this change has been that the most important measurements are expected to be made close to the Higgs production threshold.

Japan has expressed interest in hosting the ILC since several years ago but no conclusive decision has been made. In Japan's input to the Strategy Update ("Japan's Updated Strategy for Future Projects in Elementary Particle Physics" prepared by the Japan Association of High Energy Physicists (JAHEP) [9]) one reads: "With the discovery of the $125 \mathrm{GeV}$ Higgs boson at the LHC, construction of the International Linear Collider (ILC) with a collision energy of $250 \mathrm{GeV}$ should start in Japan immediately without delay".

The European Strategy Group had asked Japan to come up with a clear statement as to its commitment by the end of 2018 so that it could be considered in the Strategy Update. However, 
in mid-December 2018 it became clear that no such statement could be expected in the short term. On December 19, Japanese media published an article entitled "Science Council not to support construction of International Linear Collider in Japan" [10] and saying: "The Science Council of Japan (SCJ) compiled a draft opinion on Dec. 19 in favor of not supporting a plan to invite to the country the International Linear Collider (ILC), massive experimental equipment for the exploration of the mysteries of the universe, saying that the collider's expected scientific findings would not justify its huge construction and maintenance costs."

While SCJ is only an advisory body and decisions are to be made by Japan's Ministry of Education, Culture, Sports, Science and Technology (MEXT), the SCJ's opinion obviously had an impact. Western media reported on the subsequent developments as follows [11]:

\section{Japanese government punts on decision to host the International Linear Collider}

By Dennis Normile I Mar. 7, 2019, 12:20 PM

The government of Japan finally said something about hosting the International Linear Collider (ILC): It still can't make up its mind, and it may hold off on a decision until the fall, if not longer.

This morning in Tokyo, an official of Japan's Ministry of Education, Culture, Sports, Science and Technology (MEXT) explained to a meeting of the International Committee for Future Accelerators (ICFA) and the Linear Collider Board that the ministry could "not yet" indicate the intention of "hosting the ILC in Japan," according to a written executive summary of the presentation obtained by ScienceInsider. "MEXT will continue to discuss the ILC project with other governments while having an interest in the ILC project," the summary concludes.

"There was disappointment" among the scientists at the meeting, ICFA chair Geoffrey Taylor, an experimental physicist at the University of Melbourne in Australia admitted at a briefing this evening in Tokyo. "People were hoping there would be a statement that Japan was willing to host the ILC."

Based on the developments mentioned above, scientists outside Japan have divided opinions concerning the prospects for building the ILC in Japan. For the attitude of Japanese scientists also see the presentation given at this Conference [12].

The CLIC (“Compact Linear Collider") project has been pursued at CERN for many years. A site has been proposed that would allow the project to make use of some of the infrastructure existing at CERN (see Fig.2).

The acceleration scheme is based on using a drive beam and aims for an acceleration gradient of up to $100 \mathrm{MV} / \mathrm{m}$ (megavolts per meter). The ultimate energy goal has been $3 \mathrm{TeV}$ but a construction in stages has been envisaged as shown in Table 1 [13]. An alternative, more traditional klystron option has been proposed for the first $(380-\mathrm{GeV})$ stage but is disfavored due to its higher cost. 


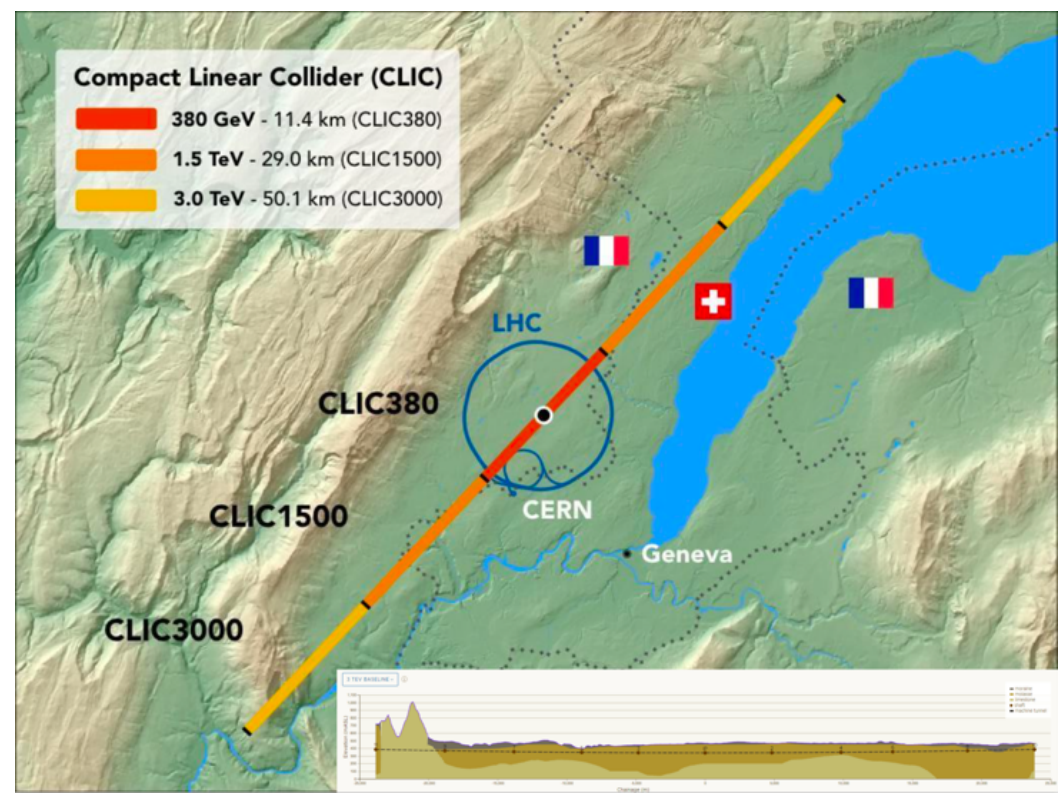

Figure 2: Possible sites for the construction of the various stages of the Compact Linear Collider (CLIC).

\begin{tabular}{|l|l|l|}
\hline Collision energy & Power consumption & Price \\
\hline $380 \mathrm{GeV}$ & $168 \mathrm{MW}$ & $5.9 \mathrm{GCHF}$ \\
$1500 \mathrm{GeV}$ & $364 \mathrm{MW}$ & $+5.1 \mathrm{GCHF}$ \\
$3000 \mathrm{GeV}$ & $589 \mathrm{MW}$ & $+7.3 \mathrm{GCHF}$ \\
\hline
\end{tabular}

Table 1: Options for the staged implementation of the Compact Linear Collider (CLIC).

\subsubsection{Circular electron-positron colliders}

Compared to linear colliders as the ones described above, circular $e^{+} e^{-}$colliders suffer from high synchrotron radiation losses but offer the advantage of higher luminosity (in particular at moderate collision energies) and of being able to accommodate more than one experiment while in a linear collider only one detector can occupy the interaction region at a given time. The high achievable luminosity at moderate energies would offer the additional advantage of enabling measurements of bosons at the $Z, W^{+} W^{-}$and $Z H$ thresholds with extremely high precision, thus yielding far better constraints for the Standard Model than the data available from LEP (see Fig.3).

Another advantage of circular $e^{+} e^{-}$colliders is that at a later time the same tunnel and the infrastructure can be reused to house a hadron collider and thus reach significantly higher collision energies, just as the LHC was built in the former LEP tunnel. The arguments most frequently put forward against circular colliders are the fact that polarized beams are hard to achieve and that the tunnel length cannot be increased to go to higher energies at a later date. Proponents of circular $e^{+} e^{-}$colliders sometimes offer the counter-argument that we know from LHC data that there is no physics in close reach for $e^{+} e^{-}$colliders beyond the tt (top-antitop) production threshold.

Projects discussed within the framework of the Strategy Update are the "Future $e^{+} e^{-}$Circular Collider (FCC-ee)" with a 100-km long tunnel at CERN (see Fig. 4 and the somewhat similar 
"Circular Electron-Positron Collider (CEPC)" in China. Both aim for collision energies above the Higgs threshold (up to $365 \mathrm{GeV}$ for FCCee and $240 \mathrm{GeV}$ for CEPC). Prices quoted range between US \$ 4.3-5 billion for CEPC [14] and 10.5 billion CHF for FCCee [15].

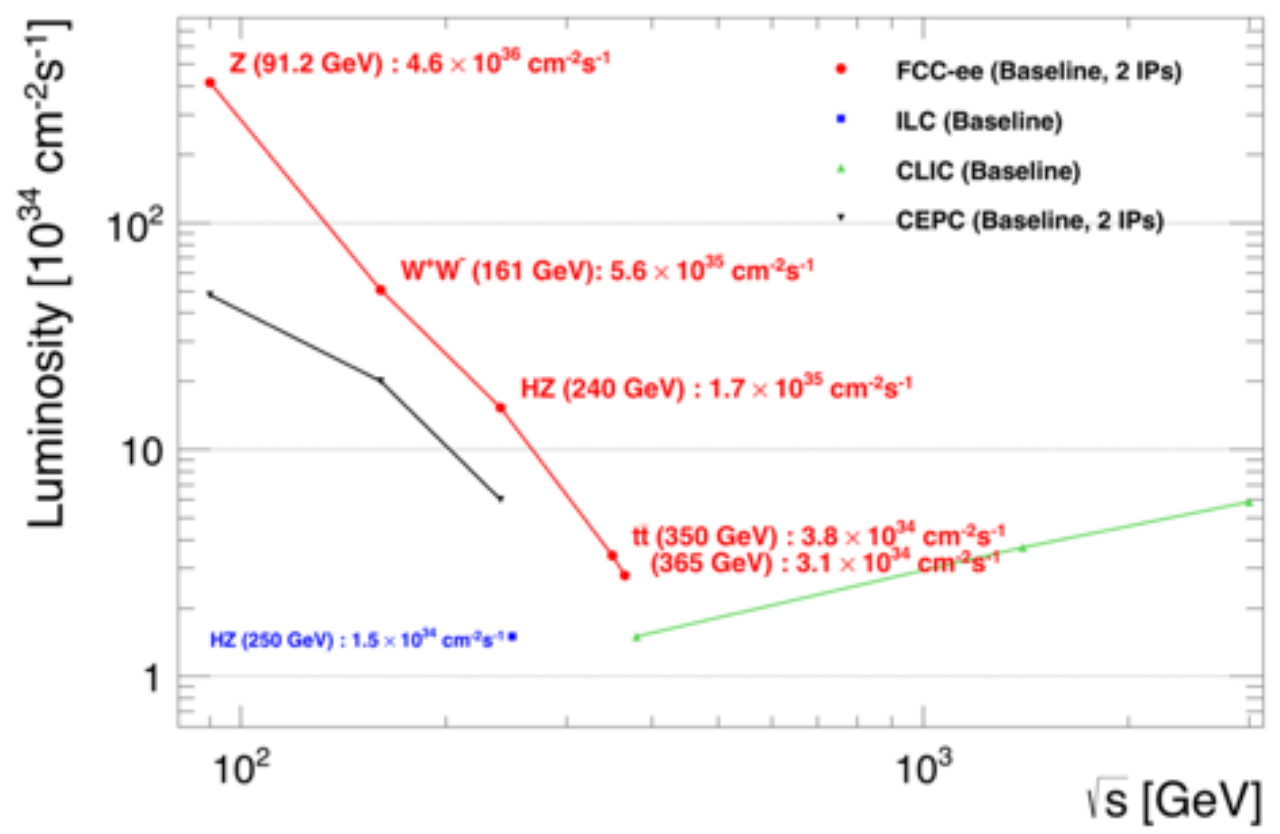

Figure 3: Luminosities to be achieved by different $e^{+} e^{-}$colliders against collision energy.

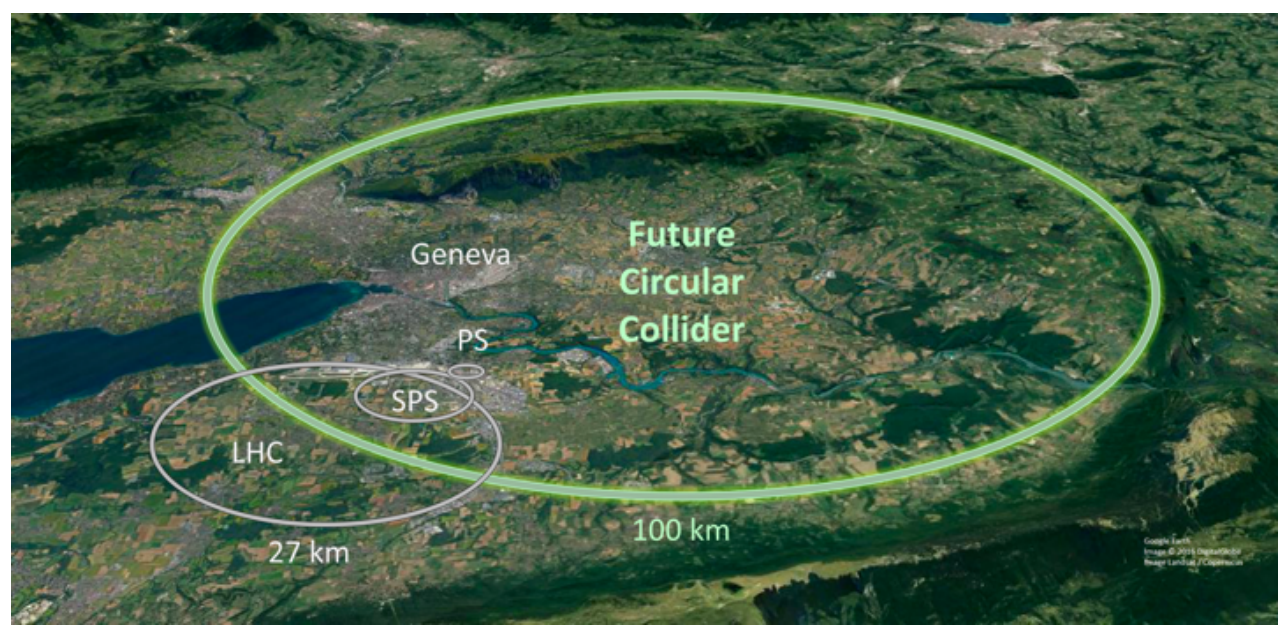

Figure 4: Possible location of the Future Circular Collider (FCC) near CERN.

\subsection{Hadron colliders}

There is widespread agreement in the community that direct discoveries of New Physics in the foreseeable future are most likely to be made at proton colliders. If a circular $e^{+} e^{-}$collider is built 
first as a Higgs factory, costs can be saved by reusing its tunnel and much of the infrastructure at a later date for a hadron collider. Differently from circular $e^{+} e^{-}$colliders, the limiting factor for the energy achievable in a circular proton collider is not synchrotron radiation but the strength of the bending magnets. In case 16-Tesla dipole magnets become available, a proton-proton collider housed in the 100-km FCC tunnel could reach a collision energy of about $100 \mathrm{TeV}$. Currently efforts are underway to reach such fields by using $\mathrm{Nb}_{3} \mathrm{Sn}$ technology but it seems that a fair amount of R\&D will be needed to reach this target. At CERN studies are underway for the "FCC-hh" collider while in China similar considerations are directed at converting the CEPC $e^{+} e^{-}$collider into the "SppC" proton collider at some future date.

Such hadron colliders should be able to collect an integrated luminosity of about $30 \mathrm{ab}^{-1}$ over their lifetime and give conclusive answers to questions such as the existence of WIMPs (Weakly Interacting Massive Particles), Higgs self-coupling, electroweak symmetry breaking phase transitions, leptoquarks and $Z^{\prime}$ bosons. In addition, they could also be used to produce proton-electron collisions at a collision energy of $3.5 \mathrm{TeV}$ ("FCC-eh") as well as heavy-ion collisions.

In case a 100-km tunnel is not within financial reach but 16-Tesla magnets can be built, an intermediate solution could be to put such magnets into the present LHC tunnel to produce a collision energy of $27 \mathrm{TeV}$. However, this would still be an expensive project (with a cost of about 7.2 billion CHF) and it could be hard to fit the new, stronger magnets into the LHC tunnel. Therefore, this "High-Energy LHC (HE-LHC)" project is not favored at the moment.

\subsection{Muon collider}

Compared to an $e^{+} e^{-}$collider a muon collider would suffer much less from synchrotron radiation due to the 200 times higher mass of the muon. At the same time, muons differ from protons in being elementary particles so that the same production cross sections for new heavy particles to be found could be achieved at much lower $\mu^{+} \mu^{-}$collisions energies than for protons (cf. Fig. 5), which would relax the requirements on bending magnet strength and/or accelerator radius. The energy reach of a muon collider of $14 \mathrm{TeV}$ collision energy would be comparable to that of a $100-\mathrm{TeV}$ proton collider.

Obviously, the fact that muons are unstable particles creates major challenges: not only will their production cost a lot of energy but their decay will create major radiation problems for the accelerator and detectors, resulting also in a signal that is much less clean than in an $e^{+} e^{-}$collider. In order to arrive at a useable number of collisions, the process from production to cooling, acceleration and collision will have to be very fast. In order to compete with a hadron collider, a muon collider would obviously have to deliver both sufficient collision energy and luminosity.

These challenges make the muon collider rather a long-term project compared to the other types of colliders discussed above. However, work is ongoing and an overview over the present state of developments can be obtained from the materials of a recent workshop [17] conducted at CERN and the paper submitted for the Strategy Update [18]. Major muon collider R\&D efforts have been undertaken within the framework of the Muon Ionization Cooling Experiment (MICE) at Rutherford-Appleton Laboratory (RAL, UK), the Muon Accelerator Program (MAP, USA) and the Low Emittance Muon Accelerator project (LEMMA, Italy). The latter project aims at reducing the time and effort needed for muon cooling by producing the muons not from the decay of pions produced in a proton accelerator but rather from the interaction of $e^{+} e^{-}$pairs creating $\mu^{+} \mu^{-}$pairs. 


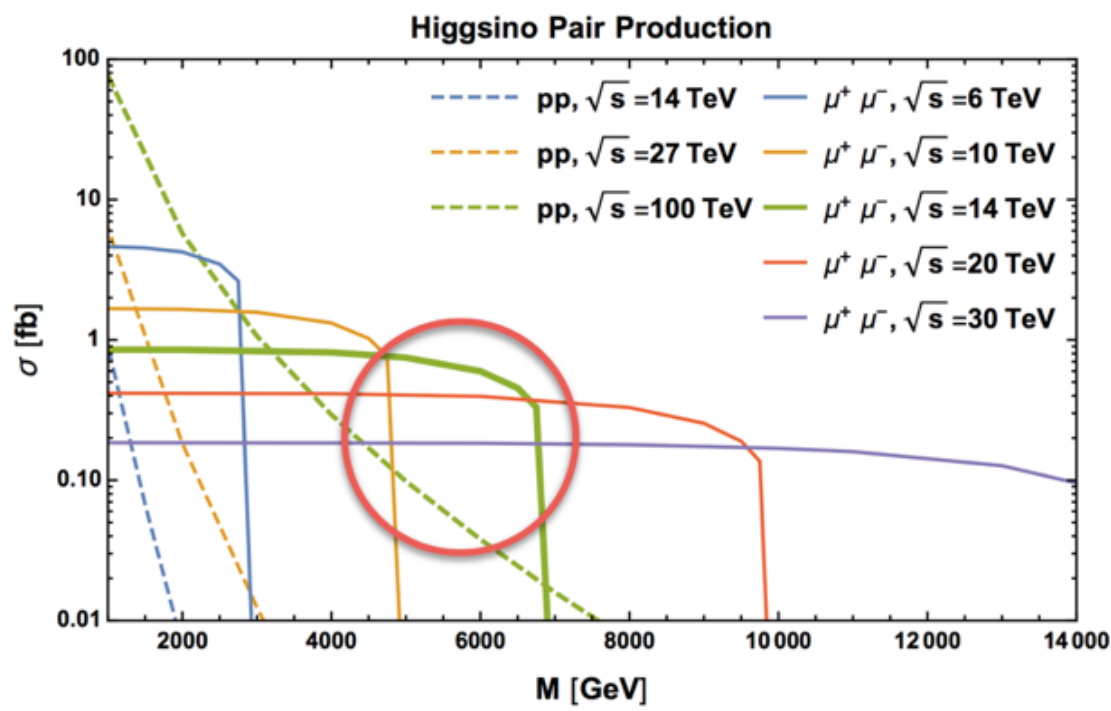

Figure 5: Calculated cross sections for the production of hypothetical Higgsino pairs at proton-proton colliders and at $\mu^{+} \mu^{-}$colliders.

Fig. 6 gives an overview over these options, which are also closely related to ideas for a neutrino factory. Note the larger acceleration rings (where magnets will have to be ramped up during the acceleration cycle) and the smaller collider rings (where a smaller circumference results in a larger number of $\mu^{+} \mu^{-}$collisions during the particles' lifetime).

\section{Non-collider projects}

Apart from ideas for new colliders, numerous other projects are being discussed for the European Strategy Update [5]. Many of them are directed at the search for various forms of dark-matter candidates such as heavy neutral leptons, axion-like particles and dark scalers or at neutrino studies. Some of these projects show synergies with colliders in that they are planned as beam-dump experiments at their injectors (such as the SHiP experiment [19], to name just one example, which could be installed within the framework of a beam dump facility to be built at the CERN SPS).

\section{Conclusion}

Since the time when contributions were submitted towards the end of 2018, many discussions have been ongoing before, during and after the Granada meeting [6]. In order to formulate a consistent joint strategy it will be important for all these discussions to converge in the near future.

Later in 2019 a "Physics Briefing Book" summarizing all the relevant information should be published. In January 2020 a Strategy Update drafting session will be conducted in Bad Honnef (Germany). The European Strategy Update is then to be submitted to the CERN Council in March 2020 so that it can be approved by Council in May 2020. 


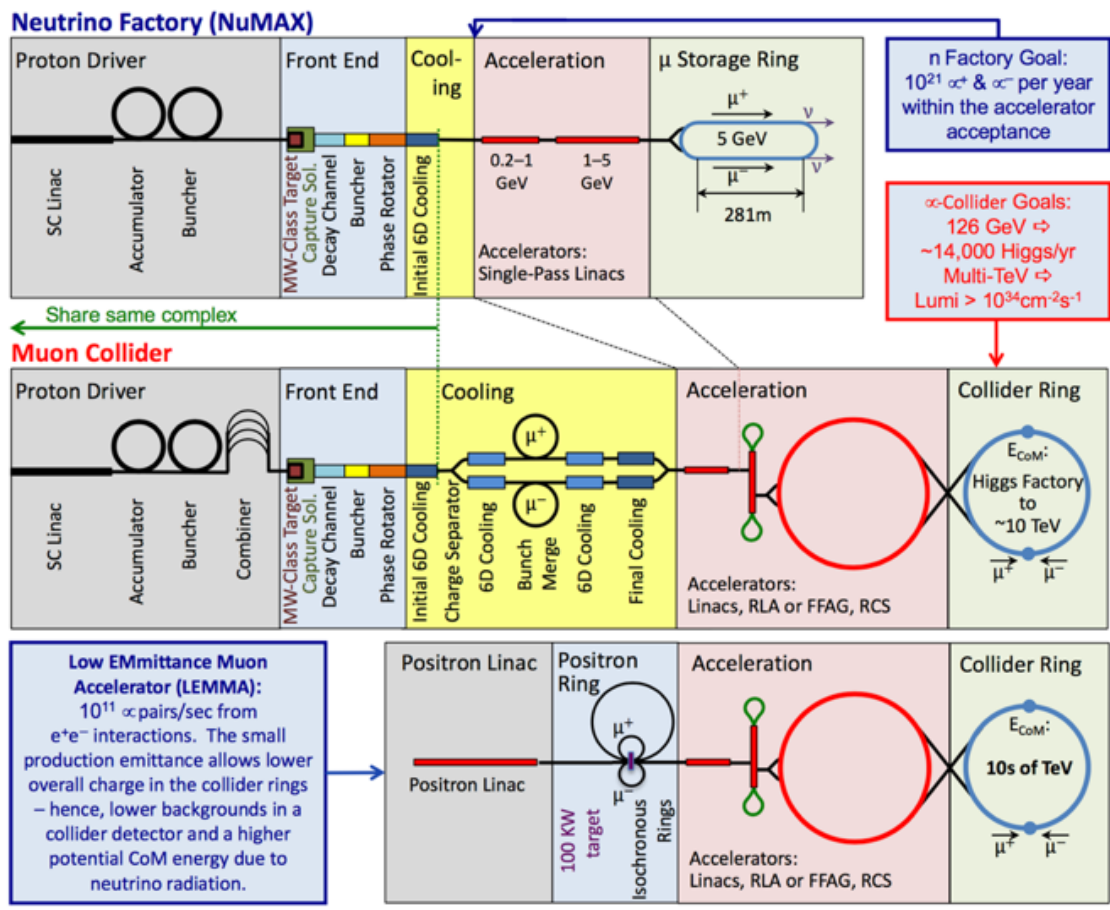

Figure 6: Different options for $\mu^{+} \mu^{-}$colliders and a neutrino factory.

\section{References}

[1] https://events.lal.in2p3.fr/conferences/Symposium06

[2] https://espp2012.ifj.edu.pl

[3] https://europeanstrategy.cern

[4] http://europeanstrategyupdate.web.cern.ch

[5] https://indico.cern.ch/event/765096/contributions

[6] https://indico.cern.ch/event/808335

[7] https://indico.cern.ch/event/789524

[8] https://indico.cern.ch/event/759130/contributions/3148649

[9] https://indico.cern.ch/event/765096/contributions/3295672/

[10] https://mainichi.jp/english/articles/20181219/p2a/00m/0na/028000c

[11] https://www.sciencemag.org/news/2019/03/japanese-government-punts-decision-host-internationallinear-collider

[12] Mihoko Nojiri, "Future colliders, expectations and reality". Presentation given at this Conference (Alps 2019)

[13] https://indico.cern.ch/event/789524/contributions/3340269

[14] https://www.nature.com/articles/d41586-018-07492-w http://english.cas.cn/newsroom/news/201810/t20181023_199981.shtml 
[15] https://indico.cern.ch/event/765096/contributions/3298137

[16] https://indico.cern.ch/event/765096/contributions/3298184/

[17] https://indico.cern.ch/event/801616

[18] https://arxiv.org/abs/1901.06150

[19] https://indico.cern.ch/event/765096/contributions/3295520 